\title{
Editorial
}

\section{Revisão das orientações metodológicas de estudos de avaliação económica de medicamentos em Portugal}

\author{
João A. Pereira \\ NOVA National School of Public Health, NOVA University Lisbon, Lisbon, Portugal
}

\section{Palavras chave}

Avaliação económica • Orientações metodológicas ·

Medicamentos

\section{Revising Methodological Guidelines for the Economic Evaluation of Pharmaceuticals in Portugal}

\section{Keywords}

Economic evaluation - Methodological guidelines ·

Pharmaceuticals

Em vários países pelo mundo fora, estudos de avaliação económica são usados para informar o processo de decisão sobre novos produtos farmacêuticos e outras tecnologias da saúde, sobretudo a questão do seu financiamento por sistemas públicos. As autoridades desses países normalmente emitem orientações metodológicas sobre a elaboração dos estudos, sendo as orientações genericamente muito semelhantes entre países com diferenças apenas nos detalhes [1].

Em Portugal, o Infarmed - Autoridade Nacional do Medicamento e Produtos de Saúde, IP (Infarmed) publicou em 1999 as Orientações Metodológicas para Estudos de

\section{KARGER}

E-Mail karger@karger.com www.karger.com/pjp

Karger Open access
(C) 2018 The Author(s). Published by S. Karger AG, Basel on behalf of NOVA National School of Public Health

This article is licensed under the Creative Commons AttributionNonCommercial-NoDerivatives 4.0 International License (CC BY NC-ND) (http://www.karger.com/Services/OpenAccessLicense) Usage and distribution for commercial purposes as well as any distribution of modified material requires written permission.
Avaliação Económica de Medicamentos [2, 3]. Com o passar do tempo este documento revelou-se algo desatualizado e a precisar de alterações. Portugal é o único país no mundo cujas Orientações remontam ao século passado e praticamente todos os países têm documentos que foram produzidos nos últimos 10 anos [4]. Ao contrário do que tem acontecido noutros países, onde as agências governamentais de avaliação de tecnologias procedem frequentemente a revisões das suas orientações [p.ex. 5-7], o documento nacional não foi ainda sujeito a revisão. No entanto, sabe-se que o Infarmed está neste momento num processo de revisão das Orientações. Seria importante que, no mínimo, fossem consideradas as alterações enunciadas a seguir.

$\mathrm{O}$ documento nacional em vigor é constituído por quinze orientações que focam questões relativas ao desenho dos estudos (p.ex. a perspetiva de análise e as fontes de dados), aspetos próprios da análise económica (p.ex. o tipo de estudo admitido, a medição e valorização de custos e a análise de sensibilidade) e aspetos processuais (p. ex. a forma de apresentação dos resultados) [8]. Resumidamente, as Orientações propõem as seguintes abordagens nos estudos de avaliação económica [2].

1 Perspectiva de análise: Deve ser a da sociedade. Desagregada em outras perspetivas relevantes, com ênfase na perspetiva do terceiro pagador. 
2 Fontes de dados: Privilegiados os resultados obtidos a partir de ensaios clínicos metodologicamente válidos com relevância para o país. Admitem-se também outras fontes de dados.

3 Comparadores: A alternativa de referência deve ser a prática corrente, ie. a terapêutica mais comum. Se não coincidir com a mais eficaz e mais barata, estas também devem ser usadas como comparadores.

4 População em estudo: O grupo populacional deve ser o que mais se aproxima da população potencialmente utilizadora da terapêutica em análise.

5 Avaliação do efeito terapêutico: Avaliado, sempre que possível, em termos de efetividade. $\mathrm{Na}$ sua falta aceitam-se dados de eficácia, sendo os modelos utilizados para estimar a efetividade completamente descritos e os resultados sujeitos a análise de sensibilidade.

6 Horizonte temporal: Período de tempo que coincida com a duração da terapêutica e das suas consequências. A utilização de modelos é admissível desde que devidamente justificada.

7 Técnicas de análise: Análise de minimização de custos (AMC), análise de custo-efetividade (ACE), análise de custo-utilidade (ACU) e análise de custo-benefício (ACB), com preferência para a análise de custo-utilidade.

8 Identificação de custos: Devem ser incluídos todos os custos relevantes para a análise. Deve ser apresentada uma árvore de decisão clínica. Se a perspetiva for a da sociedade, devem-se incluir custos diretos e indiretos, relatados separadamente. Os custos indiretos devem dizer respeito apenas às perdas de produtividade.

9 Medição e valorização dos custos: Informação sobre recursos utilizados (medidos em unidades físicas) e a forma como são valorizados (preços ou custos unitários) deve ser apresentada separada e detalhadamente e basear-se na prática clínica nacional. Deve ser iniciado um processo de criação, validação e manutenção de tabelas de custos para estudos de avaliação económica de medicamentos.

10 Medição das consequências: A unidade de medida deve ser claramente identificada. $\mathrm{Na} \mathrm{ACE}$, as consequências podem ser medidas através de vários indicadores, designadamente anos de vida ganhos, resultados intermédios, etc. Na ACU, devem ser apresentados os ponderadores da qualidade de vida e os anos de vida ganhos, e a agregação deve ser feita de forma transparente. $\mathrm{Na} \mathrm{ACB}$, privilegia-se o método da valorização contingente.

11 Análise incremental e total: Custos e consequências devem ser apresentados em termos de variação relativa- mente aos da prática clínica corrente. Devem também ser calculados os respectivos valores totais.

12 Taxa de actualização: Todos os custos e consequências devem ser actualizados a uma taxa de 5\%. Esta taxa deve ser sujeita a análise de sensibilidade. Na ACE e ACU a análise de sensibilidade deverá incluir a taxa zero no que diz respeito às consequências.

13 Avaliação do impacto da incerteza: Deve ser avaliada a sensibilidade dos resultados. No caso de valores obtidos por amostragem, a análise deverá ser feita considerando os intervalos de confiança. Noutros casos, a escolha dos intervalos de variação deve ser justificada detalhadamente com base na evidência empírica ou na lógica.

14 Modelo de referência para apresentação dos estudos: A apresentação dos estudos de avaliação económica deverá obedecer a um formulário próprio que contenha a identificação de todos os aspetos relevantes para a compreensão e análise dos estudos.

15 Aspectos éticos e de procedimento: Na apresentação do estudo, deve ser feita referência à fonte de financiamento e à contribuição real de todos os autores para a sua elaboração. Os investigadores devem ter total independência quanto à metodologia a adotar e ter o direito de publicar os resultados numa revista científica de sua escolha.

As Orientações foram preparadas para cumprir a função regulamentar de apoio aos pedidos de comparticipação de medicamentos. Ao fornecerem um modelo e uma estrutura de análise tiveram um importante efeito pedagógico para a elaboração de estudos num domínio onde havia escassez de experiência e de especialistas. No entanto, é notório que alguns dos métodos aplicados no domínio da avaliação económica em saúde sofreram grandes alterações desde que as orientações nacionais foram publicadas [9]. Exemplos de áreas onde houve desenvolvimentos científicos que devem ser incorporados numa futura revisão são:

- os métodos de extração e síntese de informação clínica e económica, tais como técnicas de revisão sistemática e de meta-análise $[10,11]$, ou de comparações indiretas e meta-análise em rede [12];

- as técnicas de modelização matemática para incorporar e extrapolar informação clínica, epidemiológica, económica e sobre qualidade de vida [13];

- os processos de análise de sensibilidade, particularmente em termos probabilísticos, praticamente desconhecidos à data da publicação das Orientações [1416]; e
II

Port J Public Health 2018;36:I-IV DOI: $10.1159 / 000495740$
Pereira 
- os instrumentos de medição de resultados em saúde, onde são vários os avanços que têm sido efectuados $[17,18]$.

Adicionalmente, há questões que foram insuficientemente tratadas nas Orientações mas que merecem hoje um tratamento mais explícito, como por exemplo:

- os métodos de medição do impacto orçamental [19, 20];

- a consideração da preferência temporal em estratégias de prevenção, como no caso da vacinação [21, 22];

- os métodos de medição de custos diretos frequentemente ignorados, tais como o tempo dos doentes e familiares, tempo de enfermagem e outros [23, 24]; e

- a adaptação de resultados de estudos de avaliação económica internacionais [25].

Finalmente, há questões particularmente importantes para a política de saúde que foram ignoradas pelas Orientações de 1999 e que deveriam merecer hoje um tratamento actualizado:
- o impacto da comparticipação do Estado sobre a equidade de acesso, financiamento e resultados [26-28];

- casos especiais como medicamentos orfãos, situações de fim de vida e outras $[29,30]$;

- a aplicação a outras tecnologias como vacinas e dispositivos médicos, e até, genericamente, a programas e intervenções em saúde [21, 27, 31-33].

Espera-se que, num futuro próximo, a revisão da Orientações do Infarmed possa incorporar os desenvolvimentos científicos identificados atrás e que as novas preocupações em política de saúde sejam devidamente enquadradas. Portugal foi pioneiro na Europa ao adotar, em 1999, critérios rigorosos de avaliação económica na decisão sobre comparticipação de medicamentos. A actualização das orientações metodológicas colocará o País, mais uma vez, na linha da frente da aplicação prática destas metodologias.

\section{References}

1 Hjelmgren J, Berggren F, Andersson F. Health economic guidelines-similarities, differences and some implications. Value Health. 2001 May-Jun;4(3):225-50.

2 Silva EA, Pinto CG, Sampaio C, Pereira JA, Drummond $M$, Trindade R. Orientações metodológicas para estudos de avaliação económica de medicamentos. Lisboa: Infarmed. Ministério da Saúde; 1999.

3 Despacho no 19 064/99. D.R. 2a Série. 233 (1999-12-09) 14893-14895.

4 ISPOR. Pharmacoeconomic guidelines around the world. Lawrenceville, NJ: ISPORThe Professional Society for Health Economics and Outcomes Research; 2018. Accessed 1 February 2018. Available from: https://tools. ispor.org/peguidelines

5 CCOHTA. Guidelines for the economic evaluation of health technologies: Canada. 4th ed. Ottawa: Canadian Agency for Drugs and Technologies in Health; 2017.

6 NICE. The guidelines manual. London: NICE-National Institute for Health and Clinical Excellence; 2012.

7 Brasil, Ministério da Saúde. Diretrizes metodológicas: diretriz de avaliação econômica. $2^{\text {a }}$ edição. Brasília: Ministério da Saúde; 2014.

8 Pereira J, Barbosa C. Avaliação económica aplicada aos medicamentos. In: Pereira J, coord. Farmacoeconomia: princípios e métodos. Madrid: Wolters Kluwer Health Pharma Solutions España; 2009. (Pharmacoeconomics SRA Formação Contínua).
9 Drummond M. Twenty years of using economic evaluations for reimbursement decisions. York: Centre for Health Economics. University of York; 2012. (CHE Research Paper; 75).

10 Vaz-Carneiro A. Cuidados de saúde baseados na evidência e nas normas de orientação clínica. In: Campos L, Borges M, Portugal R, coord. Governação dos hospitais. Alfragide: Casa das Letras; 2009. pp. 57-74.

11 Galvão TF, Pansani T, Harrad D. Principais itens para relatar revisões sistemáticas e metaanálises: a recomendação PRISMA. Epidemiol Serv Saude. 2015;24(2):335-42.

12 Hoaglin DC, Hawkins N, Jansen JP, Scott DA, Itzler R, Cappelleri JC, et al. Conducting indirect-treatment-comparison and networkmeta-analysis studies: report of the ISPOR Task Force on Indirect Treatment Comparisons Good Research Practices: part 2. Value Health. 2011 Jun;14(4):429-37.

13 Petrou S, Gray A. Economic evaluation using decision analytical modelling: design, conduct, analysis, and reporting. BMJ. $2011 \mathrm{Apr}$; 342: 11766.

14 Sculpher M, Claxton K. Establishing the costeffectiveness of new pharmaceuticals under conditions of uncertainty - when is there sufficient evidence? Value Health. 2005 Jul-Aug; 8(4):433-46.

15 Briggs A, Claxton K, Sculpher M. Decision modelling for health economic evaluation. Oxford: Oxford University Press; 2006.
16 Jain R, Grabner M, Onukwugha E. Sensitivity analysis in cost-effectiveness studies: from guidelines to practice. Pharmacoeconomics. 2011 Apr;29(4):297-314.

17 Gray AM, Clarke PM, Wolstenholme JL, Wordsworth S. Applied methods of cost-effectiveness analysis in health care. Oxford: Oxford University Press; 2011.

18 Rowen D, Brazier J. Health utility measurement. In: Glied S, Smith PC, editors. The Oxford handbook of health economics. Oxford: Oxford University Press; 2011. pp. 788-813.

19 Annemans L. Budget impact analysis. In: Arnold A, editor. Pharmacoeconomics: from theory to practice. Boca Raton: CRC Press; 2009. pp. 109-18.

20 Mauskopf J. Budget impact analysis. In: Culyer AJ, editor. Encyclopedia of health economics. Amsterdam: Elsevier; 2014.

21 Bos JM, Postma MJ, Annemans L. Discounting health effects in pharmacoeconomic evaluations: current controversies. Pharmacoeconomics. 2005;23(7):639-49.

22 Pereira JA, Barbosa C, Mateus C, Standaert B. Cost-effectiveness analysis of an HPV-16/18 prophylactic cervical cancer vaccine in a setting of existing screening in Portugal: results from a mathematical model [abstract]. Value Health. 2008;11(3):A63.

23 Adam T, Koopmanschap MA, Evans DB. Cost-effectiveness analysis: can we reduce variability in costing methods? Int J Technol Assess Health Care. 2003;19(2):407-20. 
24 Mogyorosy Z, Smith P. The main methodological issues in costing health care services: a literature review. York: University of York; 2005. (CHE Research Paper; 7).

25 Drummond M, Barbieri M, Cook J, Glick HA, Lis J, Malik F, et al. Transferability of economic evaluations across jurisdictions: ISPOR Good Research Practices Task Force report. Value Health. 2009 Jun;12(4):409-18.

26 Sassi F, Archard L, Le Grand J. Equity and the economic evaluation of healthcare. Health Technol Assess. 2001;5(3):1-138.
27 Cookson R, Drummond M, Weatherly H. Explicit incorporation of equity considerations into economic evaluation of public health interventions. Health Econ Policy Law. 2009 Apr;4(Pt 2):231-45.

28 Round J, Paulden M. Incorporating equity in economic evaluations: a multi-attribute equity state approach. Eur J Health Econ. 2018 May;19(4):489-98.

29 Drummond MF, Wilson DA, Kanavos P, Ubel P, Rovira J. Assessing the economic challenges posed by orphan drugs. Int J Technol Assess Health Care. 2007;23(1):36-42.

30 NICE. Appraising life extending, end of life treatments. London: NICE-National Institute for Health and Clinical Excellence; 2008.
31 Manca A. Economic evaluation of medical devices and drugs-same or different? Value Health. 2009 Jun;12(4):401.

32 Walker DG, Hutubessy R, Beutels P. WHO Guide for standardisation of economic evaluations of immunization programmes. Vaccine. 2010 Mar;28(11):2356-9.

33 Weatherly H, Drummond M, Claxton K, Cookson R, Ferguson B, Godfrey C, et al. Methods for assessing the cost-effectiveness of public health interventions: key challenges and recommendations. Health Policy. 2009 Dec;93(2-3):85-92. 